\title{
Target complementarity for direct dark matter detection
}

\section{David G. Cerdeño}

Instiuto de Física Teórica UAM/CSIC \& Departamento de Física Teórica,

Universidad Autónoma de Madrid, 28049 Madrid, Spain

E-mail: davidg.cerdeno@uam.es

\begin{abstract}
The combined observation of dark matter in various direct detection experiments can be used to determine the phenomenological properties of WIMP dark matter: mass, spin-dependent and spin-independent scattering cross-section off quarks. In order to understand the reconstruction of dark matter parameters, the effect of uncertainties in the nuclear spin-dependent structure functions must be taken into account. Different nuclear models that describe the spin-dependent structure function of specific target nuclei can lead to variations in the reconstructed values of the DM mass and scattering cross-section that can be similar in amplitude to that of astrophysical uncertainties, especially in those cases where the spin-dependent contribution to the elastic scattering cross-section is sizable. After reviewing these effects, the idea of target complementarity is applied to the specific case of the scintillating bolometers which are currently being developed and tested by the ROSEBUD collaboration.
\end{abstract}

VIII International Workshop on the Dark Side of the Universe,

June 10-15, 2012

Rio de Janeiro, Brazil 


\section{Direct dark matter detection and reconstruction of WIMP parameters}

Direct searches of dark matter (DM) aim to observe this abundant but elusive component of the Universe by detecting its recoils off target nuclei of a detector. A large number of experiments have been taking data in the last decades or are currently under construction with this objective, leading to a very exciting present situation. The differential event rate for the elastic scattering of a WIMP with mass $m_{\chi}$ off a nucleus with mass $m_{N}$ is given by

$$
\frac{d R}{d E_{R}}=\frac{\rho_{0}}{m_{N} m_{\chi}} \int_{v_{\min }}^{v_{e s c}} v f(v) \frac{d \sigma}{d E_{R}}\left(v, E_{R}\right) d v
$$

where $\rho_{0}$ is the local WIMP density and $f(v)$ is the WIMP velocity distribution in the detector frame. In general, the WIMP-nucleus cross-section is separated into a spin-independent (SI) and a spin-dependent (SD) contribution,

$$
\frac{d \sigma}{d E_{R}}=\frac{m_{N}}{2 \mu_{N}^{2} v^{2}}\left(\sigma_{0}^{S I, N} F_{S I}^{2}\left(E_{R}\right)+\sigma_{0}^{S D, N} F_{S D}^{2}\left(E_{R}\right)\right)
$$

where $\sigma_{0}^{S I, N}$ and $\sigma_{0}^{S D, N}$ are the SI and SD WIMP-nucleus cross-sections at zero momentum transfer. $F_{S I}\left(E_{R}\right)$ and $F_{S D}\left(E_{R}\right)$ are the SI and SD form factors that account for the coherence loss which leads to a suppression of the event rate for heavy WIMPs or heavy nuclei.

Constraints are normally expressed in terms of the SI and SD components of the WIMPnucleon elastic cross-section, $\sigma^{S I}$ and $\sigma^{S D}$, respectively. To date, the most stringent constraints on $\sigma^{S I}$ are those obtained from the XENON100 data [1] $\left(\sigma^{S I} \lesssim 2 \times 10^{-8} \mathrm{pb}\right.$ for a mass around $\left.50 \mathrm{GeV}\right)$, as well as XENON10 [2] and the low-energy reanalysis of CDMS-II [3], which dominate for light WIMPs. Regarding the SD contribution, the leading bounds have been provided by XENON [4] (SD cross-section with neutrons, $\sigma^{S D, n}$ ) and COUPP [5] and PICASSO [6] (SD cross-section with protons, $\left.\sigma^{S D, p}\right)$. Larger and more sophisticated direct detection experiments are currently under development. This is the case, for example, of the SuperCDMS and XENON1T collaborations, which aim at the construction of 1 Ton scale detectors based on germanium and xenon, respectively.

If a DM signal is obtained in a direct detection experiment, the observed rate and (if the experiment provides it) the energy dependence of the differential rate (the energy spectrum) can be used to reconstruct the properties of the DM particle $[7,8,9]$. In doing this, it is crucial to include uncertainties in the nuclear form factors and in the parameters describing the DM halo. In particular, astrophysical uncertainties are known to significantly affect the reconstruction of both the mass and scattering cross section of the DM, see e.g., Ref. [10]. Similarly, uncertainties in the spin-dependent form factors can lead to a mis-reconstruction of the WIMP mass and SD scattering cross section [11].

\section{Nuclear uncertainties in the spin-dependent structure functions}

The SD contribution to the WIMP-nucleus differential cross-section in Eq. (1.2) can be expanded as a function of the WIMP couplings to the matrix elements of the axial-vector currents in protons $\left(a_{p}\right)$ and neutrons $\left(a_{n}\right)$,

$$
\left(\frac{d \sigma}{d E_{R}}\right)_{S D}=\frac{16 G_{F}^{2} m_{N}}{\pi v^{2}} \frac{(J+1)}{J}\left(a_{p}\left\langle S_{p}\right\rangle+a_{n}\left\langle S_{n}\right\rangle\right)^{2} F_{S D}^{2}\left(E_{R}\right),
$$




\begin{tabular}{|c|ccc|ccc|}
\hline & $m_{\chi}[\mathrm{GeV}]$ & $\sigma^{S I}[\mathrm{pb}]$ & $\sigma^{S D}[\mathrm{pb}]$ & $\lambda$ & $\lambda^{S I}$ & $\lambda^{S D}$ \\
\hline BM1 & 100 & $10^{-9}$ & $10^{-5}$ & 37.2 & 36.4 & 0.8 \\
BM2 & 50 & $10^{-9}$ & $10^{-5}$ & 42.1 & 41.2 & 0.9 \\
BM3 & 100 & $10^{-9}$ & $10^{-3}$ & 79.6 & 36.4 & 43.2 \\
\hline
\end{tabular}

Table 1: Phenomenological parameters defining the benchmark models. We include the predicted total number of recoil events, $\lambda$, as well as the number of events (calculated using the R-model) $\lambda^{S I}\left(\lambda^{S D}\right)$ due to SI (SD) interactions, for the experimental setup described in the text.

where $J$ is the total spin of the nucleus and $\left\langle S_{p}\right\rangle\left(\left\langle S_{n}\right\rangle\right)$ is the proton (neutron) spin averaged over the nucleus. The SD form factor $F_{S D}^{2}\left(E_{R}\right)=S\left(E_{R}\right) / S(0)$, is commonly expressed as a decomposition into isoscalar $\left(a_{0}=a_{p}+a_{n}\right)$ and isovector $\left(a_{1}=a_{p}-a_{n}\right)$ couplings,

$$
S(q)=a_{0}^{2} S_{00}(q)+a_{0} a_{1} S_{01}(q)+a_{1}^{2} S_{11}(q),
$$

where $q$ is the momentum transfer. The quantities $S_{00}(q), S_{11}(q)$ and $S_{01}(q)$ are the spin-dependent structure functions (SDSFs), and are computed using nuclear physics models, whereas the couplings $a_{p}$ and $a_{n}$ (and consequently $a_{0}$ and $a_{1}$ ) are specific of the particle physics model for DM and are computed from the diagrams describing the WIMP-nucleon interaction. The SDSF can be calculated using a shell-model (ShM) description of the atomic nucleus, but the results are dependent on the specific potential chosen to describe the interaction among nucleons and results int he literature can differ significantly. This introduces an uncertainty in the reconstruction of the WIMP parameters (in particular its mass and SD scattering cross-section) [11]. To illustrate this we will consider two benchmark points BM1, BM2 and BM3 with the total rate and energy spectrum of nuclear recoils as given in Table 1. For concreteness we consider explicitly the case $a_{p} / a_{n}=-1$, which implies looking at only the $S_{11}$ component of the SDSF.

To incorporate nuclear uncertainties we use a description of the structure functions in terms of three parameters, $S_{i j}(u)=N\left((1-\beta) e^{-\alpha u}+\beta\right)$, where $N$ acts as an overall normalization that allows us to fit the value at zero-momentum, $\beta$ controls the height of a possible tail at large momentum and $\alpha$ provides the slope of the decreasing part in the the low-momentum regime. In Ref. [11] we determined the maximum and minimum values of the three parameters $N, \alpha$ and $\beta$ for germanium and xenon targets in such a way that they contained the predictions from the existing calculations in the literature. In the case of germanium detectors, we included the calculations of Refs. $[12,13,14]$ for ${ }^{73} \mathrm{Ge}$, and the resulting range for $S_{11}(q)$ is the following: $N=[0.12,0.21]$, $\beta=[0.020,0.042]$, and $\alpha=[5.0,6.0]$. Similarly, for xenon we used Ref. [15], in which the nuclear shell model was applied to two different potentials describing the nucleon-nucleon interaction, the Bonn A [16] and Nijmegen II [17] potentials. We also include a recent result from Ref. [18] in which the so called gen5082 interaction [19] is used. For the $S_{11}$ component in ${ }^{129} \mathrm{Xe}$ we are left with the following range of parameters, $N=[0.029,0.052], \alpha=[4.2,4.7]$, and $\beta=\left[1.0 \times 10^{-3}, 7 \times 10^{-3}\right]$. Similarly, in ${ }^{131} \mathrm{Xe}$ the ranges for $S_{11}$ are $N=[0.017,0.027]$, $\alpha=[4.3,5.0]$, and $\beta=\left[4.2 \times 10^{-2}, 6.1 \times 10^{-2}\right]$.

With this input we calculate the expected number of events and differential rate in a germanium and xenon experiment. We consider a total exposure of $\varepsilon=300 \mathrm{~kg}$ yr, that could correspond to a 

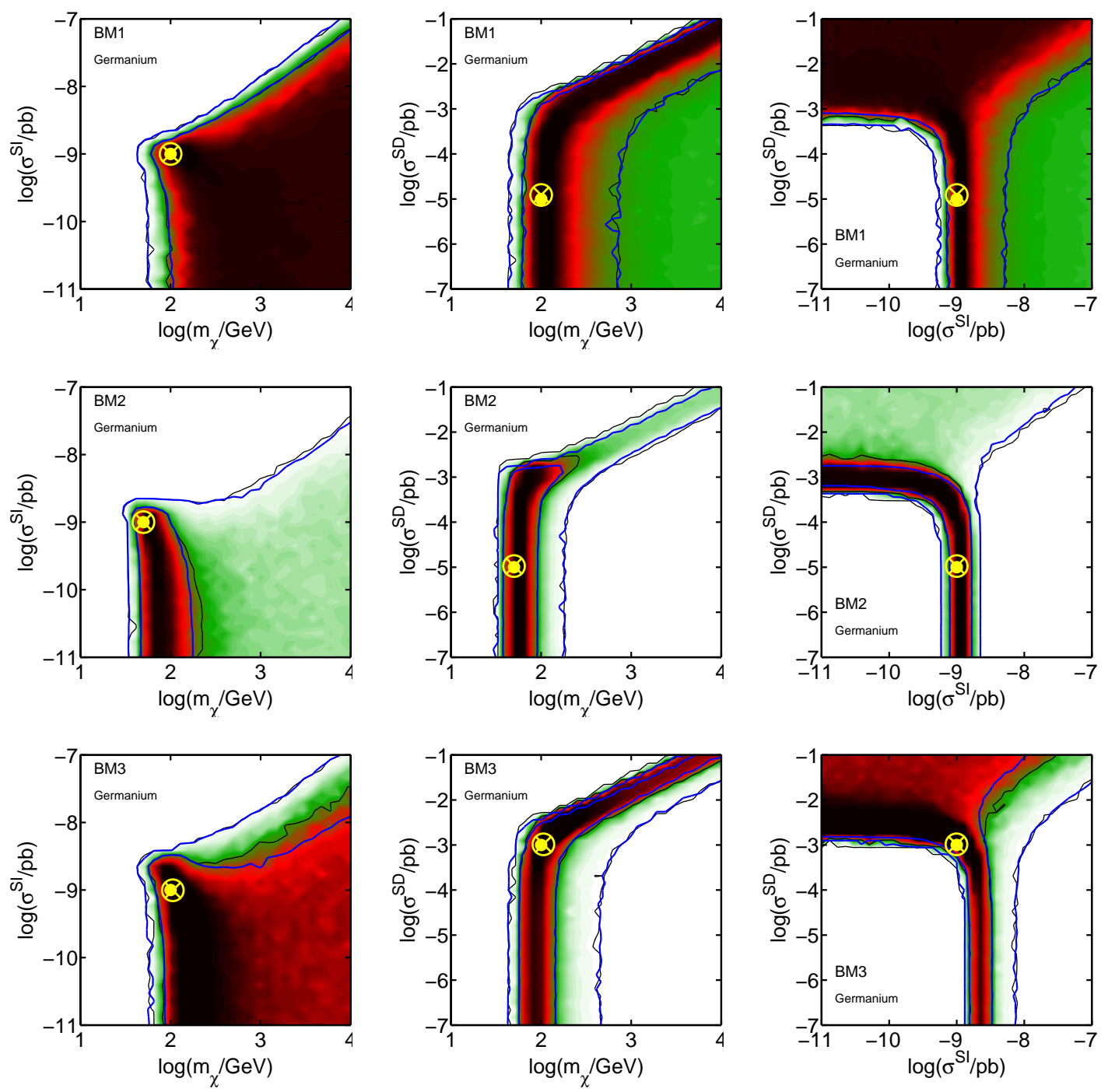

Figure 1: Two-dimensional profile likelihood for the reconstructed parameter space $\left(m_{\chi}, \sigma^{S I}, \sigma^{S D}\right)$ in benchmark models BM1, BM2 and BM3 (from top to bottom), including nuclear uncertainties in the SDSF. The inner and outer black contours are $68 \%$ and $99 \%$ confidence levels, respectively. The solid blue line corresponds to the case without uncertainties. The yellow dot indicates the benchmark value of the parameters, while the yellow encircled cross the position of the best-fit values.

1 Ton experiment operating for a whole year with an efficiency of 30\%. We consider the energy thresholds of CDMS and XENON100 for germanium and xenon, respectively and compute the expected number of dark matter events in a series of energy bins. This provides the total WIMP rate and a measurement of the recoil spectrum. We then perform a scan on the phenomenological DM parameters, including nuclear uncertainties as nuisance parameters. For each point the likelihood is calculated using the total WIMP rate and spectrum and we use a Bayesian inference algorithm, thus determining the posterior distribution function of the DM mass and scattering cross-section. The whole method is explained in detail in Ref. [11]. 
The reconstruction of DM parameters $\left(m_{\chi}, \sigma^{S I}, \sigma^{S D}\right)$ for benchmarks BM1, BM2, and BM3 are displayed in Fig. 1 in the case of a germanium detector. Black contours correspond to the results when nuclear uncertainties are taken into account, whereas blue contours correspond to the case without uncertainties. For BM1 the differences with respect to the case with no uncertainties are very small. One can only observe a slight widening in the determination of $\sigma^{S D}$ when uncertainties in the SDSF are included, but otherwise the reconstructed regions in the parameter space show very little differences. This occurs because in this point the DM candidate interacts mainly through SI interactions and it is thus fairly independent of the details of the SD term. Something similar occurs in the case of $\mathrm{BM} 2$, although the widening of the reconstruction of $\sigma^{S D}$ is more evident now. Also the $68 \%$ confidence level curves corresponding to the WIMP mass extend to slightly larger values (notice that the logarithmic scale makes this effect more difficult to observe). Finally, it is in benchmark BM3 that the largest effects are found, since the SD contribution is larger. Once more, a widening in the determination of $\sigma^{S D}$ is observed, which is now more evident in the $68 \%$ confidence level lines. Also the inclusion of uncertainties in the SDSF enlarge the contours for large WIMP masses.

The same procedure can be used for xenon detectors. Natural xenon contains two isotopes ${ }^{129} \mathrm{Xe}$ (with a $26.4 \%$ isotopic abundance) and ${ }^{131} \mathrm{Xe}(21.29 \%)$ which are sensitive to the SD component of the WIMP interaction (in particular to the SD cross-section of the WIMP with neutrons). Uncertainties in the SDSF for xenon are found to have the same qualitative effect as in germanium [11]. Despite being a heavier nucleus than germanium, the isotopic abundance of the elements sensitive to the SD coupling is larger in xenon and both effects compensate each other.

Finally, to put these results in context, we need to compare the effects of nuclear uncertainties in the SDSF that we just discussed with those originating from astrophysical uncertainties in the parameters of the DM halo. Astrophysical uncertainties affect both the reconstruction of the three DM parameters, $m_{\chi}, \sigma^{S I}$ and $\sigma^{S D}$ and are equally relevant, irrespectively of whether the main contribution comes from the SD or SI component. Nuclear uncertainties generally have a smaller effect than astrophysical ones, but they can be comparable in some benchmark scenarios, especially regarding the mass reconstruction.

\section{Complementary targets in direct detection searches}

If DM is detected, the use of different targets is crucial, as it can serve to unambiguously determine some of the WIMP properties (e.g., its interaction cross section off protons and neutrons), thus helping us discriminating among the various WIMP candidates. This idea was applied to the case of the COUPP experiment in Ref. [20], emphasizing the relevant role of targets which are sensitive to SD WIMP-nucleus interactions and showing how detection in two complementary targets (in that case $\mathrm{C}_{4} \mathrm{~F}_{10}$ and $\mathrm{C}_{3} \mathrm{FI}$ ) could allow a better measurement of the WIMP couplings. The idea of target complementarity has later been applied to the determination of the WIMP mass and cross section from different DM experiments [21,22] and the relevance of targets sensitive to the SD cross section has been analytically studied in Ref. [23]. To illustrate this, we note that, from Eq. (1.1), the total detection rate can be expressed as

$$
R=\mathscr{C}_{S I} \sigma^{S I}+\mathscr{C}_{S D} \sqrt{\sigma^{S D, p}}
$$



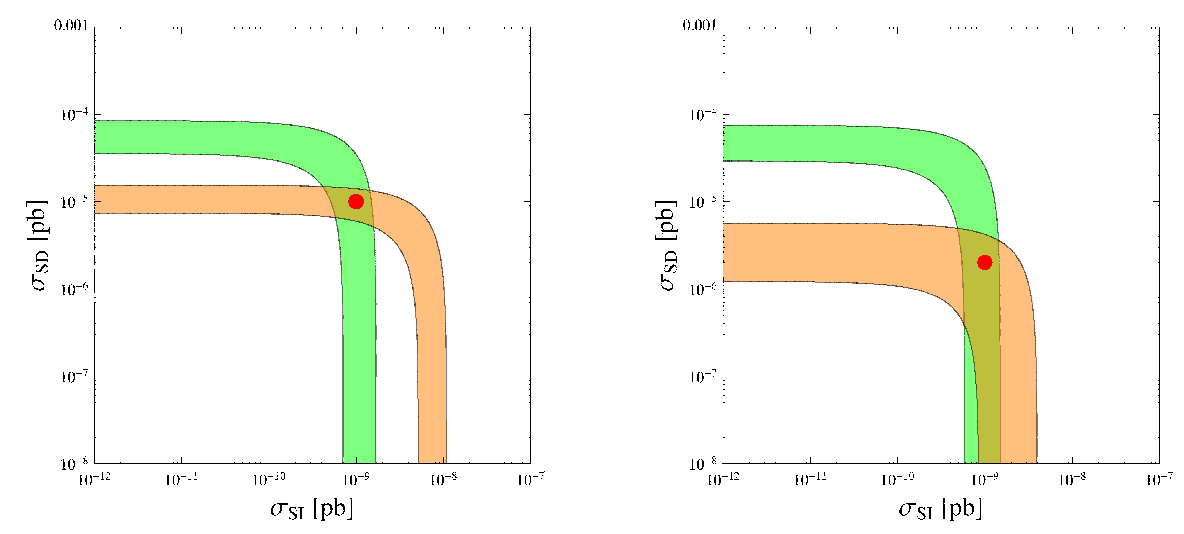

Figure 2: Schematic view of the reconstruction of the phenomenological parameters $\sigma^{S I}$ and $\sigma^{S D}$ from the observed rates in two different DM experiments (orange and green shaded areas, respectively).

where the coefficients $\mathscr{C}_{S I / S D}$ contain the integration in velocities and energies (and a dependence on the WIMP mass),

$$
\mathscr{C}_{S I / S D} \equiv \int d E_{R} \int\left(\frac{\rho_{0} f(v)}{2 \mu_{N}^{2} m_{\chi} v}\right) F_{S I / S D}^{2} d v
$$

Notice that all the dependence on the astrophysical halo parameters and the experimental setup (target material, energy threshold, energy resolution, etc.) are contained in them. Even if we assume that the WIMP mass can be determined independently with a reasonable accuracy, we are still left with two parameters to reconstruct. Thus, given only one experimental result, the same detection rate can be explained by different combinations of SI and SD couplings. This is illustrated in Fig. 2 where each of the color shaded area corresponds schematically to the region in the $\left(\sigma^{S I}, \sigma^{S D}\right)$ plane that is compatible with the detection of recoils in one particular detector, assuming the WIMP mass is known. The detection of a WIMP in a second experiment with a different target can provide complementary information with which this degeneracy can be partially resolved, since changing target implies that also the $\mathscr{C}$ coefficients in Eq. (3.1) are different. Obviously, for this to work one needs targets in a large sensitivity to SD interactions. Fig. 2 depicts two possible situations: on the left hand-side we consider an example in which two targets are complementary and allow a good reconstruction of both the SD and SI couplings. This is the case, for example, if one target is mostly sensitive to SI interactions and the other one to SD ones. On the right hand-side we show another case in which complementarity is not present since the overlapping regions is not bound from below. This is generically the case when the two targets are mostly sensitive to the SI coupling, and therefore is a very common situation.

In Fig. 3 we consider the situation in which a WIMP signal is observed in three experiments for benchmark point BM2. First we take a germanium and a xenon experiment with the characteristics of SuperCDMS and XENON1T. Then we include a hypothetical scintillating bolometer with a $\mathrm{CaWO}_{4}$ or $\mathrm{Al}_{2} \mathrm{O}_{3}$ target. The blue contours correspond to the reconstruction of DM parameters using only the data from germanium and xenon. As we can observe, the contours are not closed and none of the DM parameters can be determined with a reasonable accuracy. On the other hand, the inclusion of a target with an enhanced sensitivity to SD couplings improves this situation. In 

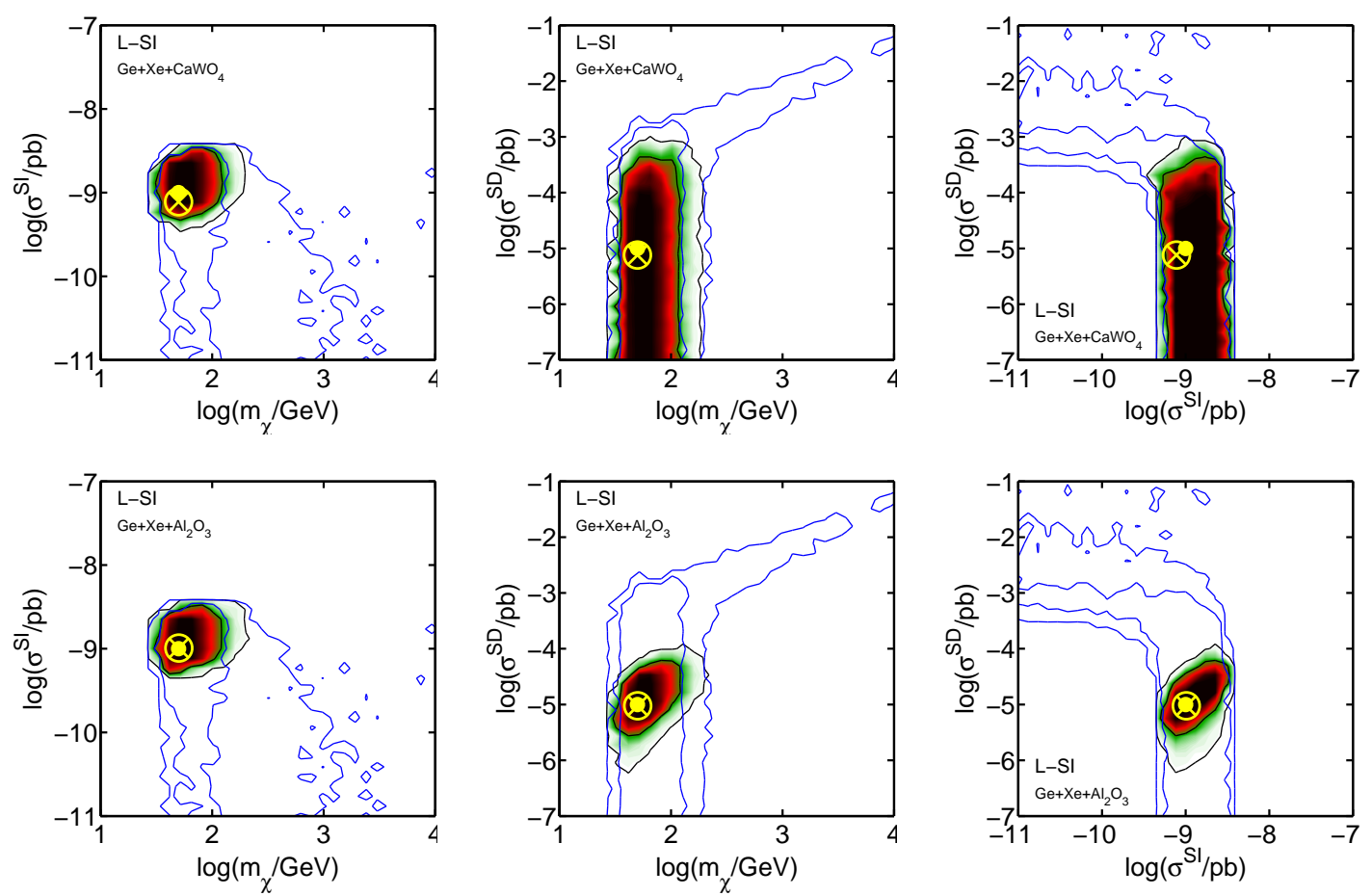

Figure 3: Profile likelihood for the dark matter parameters for the benchmark point BM2 after the combination of data from SuperCDMS, Xenon1T and a bolometric target $\left(\mathrm{CaWO}_{4}\right.$ and $\mathrm{Al}_{2} \mathrm{O}_{3}$ from top to bottom, respectively). The blue lines correspond to the case when only SuperCDMS and Xenon1T are used.

the case of $\mathrm{CaWO}_{4}$ the WIMP mass and SI independent cross-section are quite well reconstructed but only an upper limit can be obtained for the SD component. Finally, in the case of a $\mathrm{Al}_{2} \mathrm{O}_{3}$ target the three components can be identified. The reconstructing power of this method is very dependent on the actual regions of the parameter space [24].

\section{Conclusions}

We have studied the effect that uncertainties in the nuclear spin-dependent structure functions have in the reconstruction of DM properties by means of direct detection experiments. We observe that they can affect the computation of the WIMP mass and SD cross-section and that these effects can be comparable to those induced by astrophysical uncertainties, especially in those cases in which the SD contribution to the total detection rate is large.

We also introduced the idea of complementarity of direct detection experiments and applied it to several targets currently under development by the ROSEBUD collaboration. We show how, when these are used in combination with germanium and xenon experiments, some degeneracies in the determination of the phenomenological WIMP parameters can be removed.

\section{Acknowledgements}

D.G.C. is supported by the Ramón y Cajal program of the Spanish MICINN, the Consolider- 
Ingenio 2010 Programme under grant MultiDark CSD2009-00064, the Spanish MICINN under grant FPA2009-08958, the Community of Madrid under grant HEPHACOS S2009/ESP-1473, and the European Union under the Marie Curie-ITN program PITN-GA-2009-237920.

\section{References}

[1] E. Aprile et al. [XENON100 Collaboration], Phys. Rev. Lett. 109 (2012) 181301 [arXiv:1207.5988].

[2] J. Angle et al. [XENON10 Collaboration], Phys. Rev. Lett. 107 (2011) 051301 [arXiv:1104.3088].

[3] Z. Ahmed et al. [CDMS-II Collaboration], Phys. Rev. Lett. 106, 131302 (2011) [arXiv:1011.2482].

[4] J. Angle, E. Aprile, F. Arneodo, L. Baudis, A. Bernstein, A. Bolozdynya, L. C. C. Coelho and C. E. Dahl et al., Phys. Rev. Lett. 101, 091301 (2008) [arXiv:0805.2939].

[5] E. Behnke, J. Behnke, S. J. Brice, D. Broemmelsiek, J. I. Collar, P. S. Cooper, M. Crisler and C. E. Dahl et al., Phys. Rev. Lett. 106 (2011) 021303 [arXiv:1008.3518].

[6] S. Archambault et al. [PICASSO Collaboration], Phys. Lett. B 711 (2012) 153 [arXiv:1202.1240].

[7] A. M. Green, JCAP 0708, 022 (2007) [arXiv:hep-ph/0703217].

[8] A. M. Green, JCAP 0807 (2008) 005 [arXiv:0805.1704].

[9] M. Drees and C. -L. Shan, JCAP 0706 (2007) 011 [astro-ph/0703651].

[10] A. M. Green, Mod. Phys. Lett. A 27 (2012) 1230004 [arXiv:1112.0524].

[11] D. G. Cerdeño, M. Fornasa, J. -H. Huh and M. Peiro, arXiv:1208.6426.

[12] V. A. Bednyakov and F. Simkovic, Phys. Part. Nucl. 37 (2006) S106 [hep-ph/0608097].

[13] M. T. Ressell, M. B. Aufderheide, S. D. Bloom, K. Griest, G. J. Mathews and D. A. Resler, Phys. Rev. D 48 (1993) 5519.

[14] V. Dimitrov, J. Engel and S. Pittel, Phys. Rev. D 51 (1995) 291 [hep-ph/9408246].

[15] M. T. Ressell and D. J. Dean, Phys. Rev. C 56 (1997) 535 [hep-ph/9702290].

[16] M. Hjorth-Jensen, T. T. S. Kuo and E. Osnes, Phys. Rept. 261, 125 (1995).

[17] V. G. J. Stoks, R. A. M. Klomp, C. P. F. Terheggen and J. J. de Swart, Phys. Rev. C 49, 2950 (1994) [nucl-th/9406039].

[18] J. Menéndez, D. Gazit and A. Schwenk, arXiv:1208.1094.

[19] E. Caurier, J. Menendez, F. Nowacki and A. Poves, Phys. Rev. Lett. 100, 052503 (2008) [arXiv:0709.2137].

[20] G. Bertone, D. G. Cerdeño, J. I. Collar and B. C. Odom, Phys. Rev. Lett. 99, 151301 (2007) [arXiv:0705.2502].

[21] M. Drees and C. -L. Shan, JCAP 0806, 012 (2008) [arXiv:0803.4477].

[22] M. Pato, L. Baudis, G. Bertone, R. Ruiz de Austri, L. E. Strigari, R. Trotta, Phys. Rev. D83 (2011) 083505. [arXiv:1012.3458].

[23] M. Cannoni, J. D. Vergados and M. E. Gomez, Phys. Rev. D 83, 075010 (2011) [arXiv:1011.6108].

[24] D.G. Cerdeño, C. Cuesta, M. Fornasa, E. García, J.-H. Huh, M. Martínez, Y. Ortigoza, L. Robledo, M. Sarsa et al. Work in progress. 